\title{
A Redundant Parameter Based Recursive Least Squares Algorithm for Nonlinear Additive Systems with Unknown Time- Delay
}

\author{
Jia Chen ${ }^{1}$ and Donglei $\mathrm{Lu}^{2}$ \\ ${ }^{1,2}$ Wuxi Professional College of Science and Technology, Wuxi 214028, PR China \\ 1chenjia0104@163.com,229493011@qq.com
}

\begin{abstract}
In this paper, a new identification method is proposed for nonlinear additive systems with unknown time-delay. The additive systems have two different nonlinearities, one is an unknown structure nonlinear input, the other is a preload nonlinear output. The key term separation principle is utilized to simplify the two nonlinearities. Since an unknown timedelay is introduced, a redundant parameter based recursive least squares algorithm is derived. The simulation results indicate that the proposed method is effective.
\end{abstract}

Keywords: Parameter estimation, Key term separation principle, Redundant parameter, Preload nonlinearity, Additive system

\section{Introduction}

Nonlinear systems are often existed in industrial processes [1, 2]. There are two different kinds of nonlinearities, the polynomial nonlinearities and the hard nonlinearities $[3,4]$. The polynomial nonlinearities which can be written as an analytic function of the input have been received much attention. Recently, many identification methods have been proposed for system with polynomial nonlinearities, such as the stochastic gradient (SG) algorithm [5, 6], the recursive least squares (RLS) algorithm [7, 8] and the iterative algorithm $[9,10]$. Unfortunately, these methods cannot be directly used for systems with hard nonlinearities.

Hard nonlinearities in which the parameters in cascade with the linear systems are common in engineering practice [11, 12, 13]. Identification of systems with hard nonlinearities is difficult, it is of the fact that the output of the hard nonlinear block cannot be written as an analytical function of the input. The key term separation principle is often utilized to simplify the hard nonlinearities. The basic idea of the key term separation principle is first to apply some mathematical functions to turn the model of the hard nonlinear systems into an identification model and then use the linear system identification methods to estimate the unknown parameters [14, 15]. For example, Chen et al. defined switching function to simplify a saturation and dead-zone nonlinearity and proposed an identification method for the nonlinear system [16]. Jacob et al applied a direct weight optimization method for nonlinear systems with hard nonlinearities [17]. Yu et al. provided an iterative identification method for Hammerstein output-error systems with two-segment nonlinearities [18]. However, all the above works assumed that the hard nonlinearities have known structures, when the hard nonlinearities have unknown structures, these methods may not be available.

With the development of the network, researchers often use network to transmit the control signals. Due to the complexity of the network, some control signals may have time-delay which limits the performance of system control, fault detection and fault diagnosis. Clearly, system identification for time-delay systems plays a crucial part in control designs

Received (December 3, 2016), Review Result (October 18, 2017), Accepted (November 10, 2017) 
and fault detection. Recently, there exist many works for system with time-delay. Gu et al developed an iterative algorithm for state space systems with multi-state delays and a RLS algorithm canonical state space models with d-step state- delay [19, 20]. However the time delays are assumed to be known in prior. Xie et al proposed an EM algorithm for multirate processes with random delays, and all the random delays are unknown [21]. Unfortunately, all the data in each step of the EM method are applied to estimate the unknown parameters and the two steps of the EM method are iteratively operated, which makes the EM method have heavy computational effort. Now a question naturally arises: Can we provide an identification method which not only has less computational effort but also can estimate the parameters and the unknown time-delay simultaneously. To answer this question is the focus of this paper.

In this paper, we propose a redundant parameter based RLS algorithm for nonlinear additive systems with unknown time-delay. The unknown continuous nonlinear input and the preload nonlinear output can be simplified by using the key term separation principle. Then a redundant parameter based RLS algorithm is proposed to estimate the parameters and the time-delay. Briefly, the paper is organized as follows. Section 2 introduces the additive system and the hard nonlinearities. Section 3 studies an identification method for the nonlinear additive system with unknown time-delay. Section 4 provides an illustrative example. Finally, concluding remarks are given in Section 5.

\section{The Additive System and the Hard Nonlinearities}

Consider the following additive system which is shown in Figure 1,

$y(t)=B(z) f(u(t)+A(z) g(y(t-\tau))+v(t),(1)$ where $y(t)$ is the system output, $u(t)$ is the system input, $v(t)$ is a stochastic white noise with zero

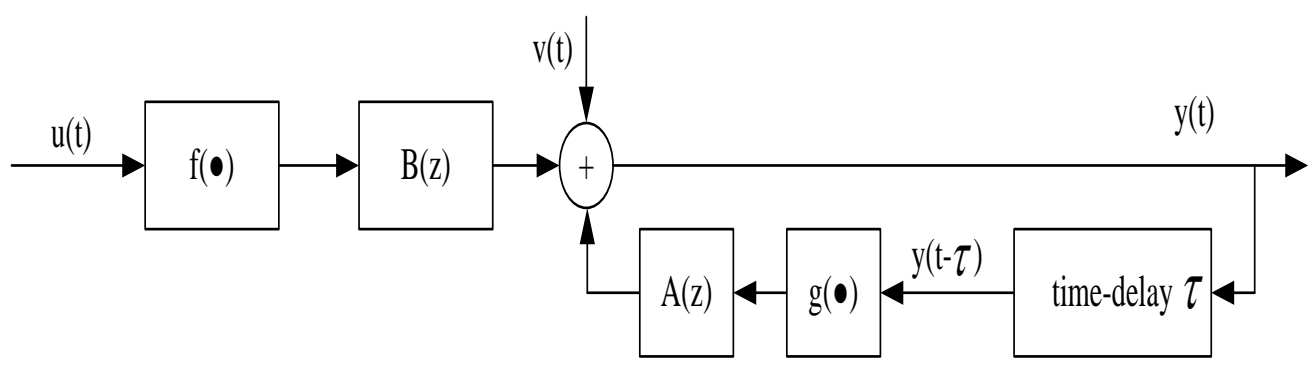

Figure 1. The Additive Nonlinear System with Time-Delay

mean, $\tau$ is an unknown time-delay, and $A(z)$ and $B(z)$ are polynomials in the unit backward shift operator $\left[z^{-1} y(t)=y(t-1)\right]$ and

$$
\begin{aligned}
& A(z):=a_{1}+a_{2} z^{-1}+\cdots+a_{n} z^{-n+1}, \\
& B(z):=b_{1} z^{-1}+b_{2} z^{-2}+\cdots+b_{n} z^{-n} .
\end{aligned}
$$




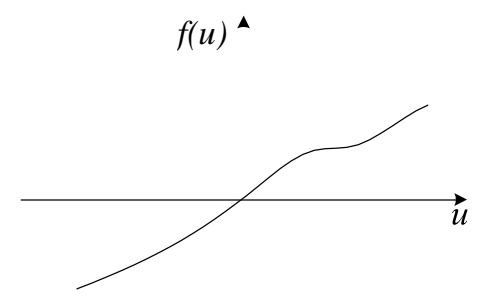

Figure 2. The Continuous Nonlinearity

The nonlinear input $f(u(t))$ has an unknown structure which is shown in Figure 2.

The nonlinear output $g(y(t-\tau))$ is a preload nonlinearity expressed in [11].

By using Weierstrass approximation theorem, the nonlinear input can be approximated by:

$f(u(t))=c_{1} u(t)+c_{2} u^{2}(t)+c_{3} u^{3}(t)+\cdots+c_{p} u^{p}(t)$.

Define a switching function

$$
\operatorname{sgn}(y(t-\tau)):= \begin{cases}1, & \text { if } y(t-\tau)>0, \\ 0, & \text { if } y(t-\tau)=0, \\ -1, & \text { if } y(t-\tau)<0 .\end{cases}
$$

Then $g(y(t-\tau))$ can be simplified as

$g(y(t-\tau))=y(t-\tau)+\frac{m_{1}+m_{2}}{2} \operatorname{sgn}(y(t-\tau))+\frac{m_{1}+m_{2}}{2} \operatorname{sgn}\left(y^{2}(t-\tau)\right)$.

Assuming

$d_{1}=\frac{m_{1}+m}{2}, \quad d_{2}=\frac{m_{1}-m_{2}}{2}$,

gives

$g(y(t-\tau))=y(t-\tau)+d_{1} \operatorname{sgn}(y(t-\tau))+d 2 \operatorname{sgn}\left(y^{2}(t-\tau)\right)$.

\section{The Identification Method}

Equation (1) can be written as

$$
\begin{aligned}
y(t)= & B(z)\left(c_{1} u(t)+c_{2} u^{2}(t)+c_{3} u^{3}(t)+\cdots+c_{p} u^{p}(t)\right)+ \\
& A(z)\left(y(t-\tau)+d_{1} \operatorname{sgn}(y(t-\tau))+d 2 \operatorname{sgn}\left(y^{2}(t-\tau)\right) .\right.
\end{aligned}
$$

Define the parameter vector $\boldsymbol{\theta}$ and the information vector $\boldsymbol{\varphi}(t)$ as

$$
\begin{aligned}
\theta:=\left[a_{1}, a_{2}, \cdots, a_{n}, a_{1} d_{1}, a_{2} d_{2}, \cdots a_{n} d_{1}, a_{1} d_{2}, a_{2} d_{2}, \cdots, a_{n} d_{2}, b_{1} c_{1}, b_{1} c_{2}, \cdots, b_{1} c_{p}, b_{2} c_{1}, b_{2} c_{2}, \cdots,\right. \\
\left.b_{2} c_{p}, \cdots, b_{n} c_{1}, b_{n} c_{2}, \cdots, b_{n} c_{p}\right]^{T} \in R^{n p+3 n}, \\
\varphi(t):=[y(t-\tau), y(t-\tau-1), \cdots, y(t-\tau-n+1), \operatorname{sgn}(y(t-\tau)), \operatorname{sgn}(y(t-\tau-1)), \cdots, \\
\quad \operatorname{sgn}(y(t-\tau-n+1)), \operatorname{sgn}\left(y^{2}(t-\tau)\right), \operatorname{sgn}\left(y^{2}(t-\tau-1)\right) \cdots, \operatorname{sgn}\left(y^{2}(t-\tau-n+1)\right), \\
u(t-1), u^{2}(t-1), \cdots, u^{p}(t-1), u(t-2), u^{2}(t-2), \cdots, u^{p}(t-2), \cdots, \\
\left.u(t-n), u^{2}(t-n), \cdots, u^{p}(t-n)\right]^{T} \in R^{n p+3 n},
\end{aligned}
$$

one has

$$
y(t)=\varphi^{T}(t) \theta+v(t)
$$


In order to separate $b_{i}$ from $b_{i} c_{j}$, we adopt the assumption that the first coefficient $b_{1}$ equals 1, i.e., $b_{1}=1$. The parameter vector $\theta$ be defined as

$$
\begin{aligned}
\theta= & {\left[a_{1}, a_{2}, \cdots, a_{n}, a_{1} d_{1}, a_{2} d_{2}, \cdots a_{n} d_{1}, a_{1} d_{2}, a_{2} d_{2}, \cdots, a_{n} d_{2}, c_{1}, c_{2}, \cdots, c_{p}, b_{2} c_{1}, b_{2} c_{2}, \cdots,\right.} \\
& \left.b_{2} c_{p}, \cdots, b_{n} c_{1}, b_{n} c_{2}, \cdots, b_{n} c_{p}\right]^{T} .
\end{aligned}
$$

If all the variables in the information vector $\varphi(t)$ are known, the following RLS algorithm can be obtained:

$$
\begin{gathered}
\hat{\theta}(t)=\hat{\theta}(t-1)+P(t) \varphi(t) e(t) \\
e(t)=y(t)-\varphi^{T}(t) \hat{\theta}(t-1) \\
P(t)=P(t-1)-\frac{P(t-1) \varphi(t) \varphi^{T}(t) P(t-1)}{1+\varphi^{T}(t) P(t-1) \varphi(t)} .
\end{gathered}
$$

Unfortunately, the unknown time-delay $\tau$ leads the variables $y(t-\tau), \operatorname{sgn}(y(t-\tau))$ unknown, thus the RLS cannot be used to estimate the parameters. Therefore, we introduce a redundant parameter based method to overcome this difficulty. Assume $M$ is sufficiently large, and satisfies $n+\tau \leq M$.

Define the parameter vector $\boldsymbol{\theta}$ and the information vector $\boldsymbol{\varphi}(t)$ as

$$
\begin{aligned}
\theta:= & {\left[a_{1}, a_{2}, \cdots, l_{\tau-1}, a_{1}, a_{2}, \cdots, a_{n}, l_{n+\tau-1}, \cdots, l_{M}, l_{1} d_{1}, l_{2} d_{1}, \cdots, l_{\tau-1} d_{1}, a_{1} d_{1}, a_{2} d_{1}, \cdots, a_{n} d_{1},\right.} \\
& l_{n+\tau-1} d_{1}, \cdots, l_{M} d_{1}, l_{1} d_{2}, l_{2} d_{2}, \cdots, l_{\tau-1} d_{2}, a_{1} d_{2}, a_{2} d_{2}, \cdots, a_{n} d_{2}, l_{n+\tau-1} d_{2}, \cdots, l_{M} d_{2}, \\
& \left.c_{1}, c_{2}, \cdots, c_{p}, b_{2} c_{1}, b_{2} c_{2}, \cdots, b_{2} c_{p}, \cdots, b_{n} c_{1}, b_{n} c_{2}, \cdots, b_{n} c_{p}\right]^{T} \in R^{n p+3 M}, \\
\varphi(t):= & y(t-1), y(t-2), \cdots, y(t-\tau+1), y(t-\tau), y(t-\tau-1), \cdots, y(t-\tau-n+1), \\
& y(t-\tau-n), y(t-\tau-n-1), \cdots, y(t-M), \operatorname{sgn}(y(t-1)), \operatorname{sgn}(y(t-2)), \cdots, \\
& \operatorname{sgn}(y(t-\tau+1)), \operatorname{sgn}(y(t-\tau)), \operatorname{sgn}(y(t-\tau-1)), \cdots, \operatorname{sgn}(y(t-\tau-n+1)), \\
& \operatorname{sgn}(y(t-\tau-n)), \operatorname{sgn}(y(t-\tau-n-1)), \operatorname{sgn}(y(t-\tau-1)), \cdots, \operatorname{sgn}(y(t-M)), \operatorname{sgn}\left(y^{2}(t-1)\right), \\
& \operatorname{sgn}\left(y^{2}(t-2)\right), \cdots, \operatorname{sgn}\left(y^{2}(t-\tau+1)\right), \operatorname{sgn}\left(y^{2}(t-\tau)\right), \operatorname{sgn}\left(y^{2}(t-\tau-1)\right), \cdots, \\
& \operatorname{sgn}\left(y^{2}(t-\tau-n+1)\right), \operatorname{sgn}\left(y^{2}(t-\tau-n)\right), \operatorname{sgn}\left(y^{2}(t-\tau-n-1)\right), \cdots, \operatorname{sgn}\left(y^{2}(t-M)\right), \\
& u(t-1), u^{2}(t-1), \cdots, u^{p}(t-1), u(t-2), u^{2}(t-2), \cdots, u^{p}(t-2), \cdots, u(t-n), \\
& \left.u(t-n), \cdots, u^{p}(t-n)\right]^{T} \in R^{n p+3 M} .
\end{aligned}
$$

Using the following RLS algorithm to estimate the parameter vector $\theta$ in (12):

$$
\begin{aligned}
& \hat{\theta}(t)=\hat{\theta}(t-1)+P(t) \varphi(t) e(t), \\
& e(t)=y(t)-\varphi^{T}(t) \hat{\theta}(t-1), \\
& P(t)=P(t-1)-\frac{P(t-1) \varphi(t) \varphi^{T}(t) P(t-1)}{1+\varphi^{T}(t) P(t-1) \varphi(t)} .
\end{aligned}
$$

Since $y(t)$ only depends on $y(t-\tau), \cdots, y(t-\tau-n+1), y(t-\tau-n), y(t-\tau-n-1), \cdots$, $y(t-M), \operatorname{sgn}(y(t-\tau)), \operatorname{sgn}(y(t-\tau-1)), \cdots, \operatorname{sgn}(y(t-\tau-n+1)), \operatorname{sgn}\left(y^{2}(t-\tau)\right), \operatorname{sgn}\left(y^{2}(t-\tau\right.$ $-1)), \cdots, \operatorname{sgn}\left(y^{2}(t-\tau-n+1)\right)$ and the input, once the parameter vector has been estimated, some irrelevant parameters such as $l 1, l 2, \cdots, l_{\tau-1}, l_{n+\tau-1}, \cdots, l M$, equal to zero. From the parameter vector $\boldsymbol{\theta}$ in (12), we can conclude that the following four vectors whose entries are all zero,

$$
\begin{aligned}
& P_{1}:=\left[l_{1}, l_{2}, \cdots, l_{\tau-1}\right]^{T}, \\
& P_{2}:=\left[l_{n+\tau-1}, \cdots, l_{M} l_{1} d_{1}, l_{2} d_{1}, \cdots, l_{\tau-1} d_{1}\right]^{T},
\end{aligned}
$$




$$
\begin{aligned}
& P_{3}:=\left[l_{n+\tau-1} d_{1}, \cdots, l_{M} d_{1}, l_{1} d_{2}, l_{2} d_{2} \cdots, l_{\tau-1} d_{2}\right]^{T}, \\
& P_{4}:=\left[l_{n+\tau-1} d_{2}, \cdots, l_{M} d_{2}\right]^{T} .
\end{aligned}
$$

According to (17), we can compute the time-delay $\tau$.

However, in application, the irrelevant parameter estimates may be very small. In order to pick out these irrelevant parameters correctly, we should give a small constant $\epsilon$ in prior. If the absolute value of an unknown parameter is smaller than $\epsilon$, we can regard the parameter as a irrelevant parameter and pick it out. The choice of the constant $\epsilon$ is critical. A small $\epsilon$ may lead some irrelevant parameters not be picked out from the parameter vector. On the other hand, a big $\epsilon$ may mistake some relevant estimates for the irrelevant parameters.

The steps of computing the parameter estimation vector $\hat{\theta}(t)$ by using the redundant parameter based RLS algorithm are listed in the following.

1. Let $\hat{\theta}(0)=1 / p_{0}$ and $P(0)=p_{0} I$ with 1 being a column vector whose entries are all unity, $\mathrm{I}$ be an identity matrix of appropriate size and $p_{0}=10^{6}$.

2. Let $t=1, y(j)=0, u(j)=0, j \leq 0$, and give two small positive numbers $\varepsilon$ and $\delta$.

3. Collect the input-output data $\{\mathrm{u}(\mathrm{t}), \mathrm{y}(\mathrm{t})\}$.

4. Form $\hat{\varphi}(t)$ by (13).

5. Compute e(t) and $\mathrm{P}(\mathrm{t})$ by (15) and (16), respectively.

6. Update the parameter estimation vector $\hat{\theta}(t)$ by (14).

7. Compare $\hat{\theta}(t)$ and $\hat{\theta}(t-1)$ : if $\|\hat{\theta}(t)-\hat{\theta}(t-1)\| \leq \delta$, then terminate the procedure and obtain the $\hat{\theta}(t)$ and go to the next step; otherwise, increase t by 1 and go to step 3.

8. Compare each parameter estimate in the parameter estimation vector $\theta \hat{\theta}(t)$ with $\varepsilon$, and pick out the irrelevant parameters.

9. Compute the unknown time-delay by (17).

\section{Example}

Consider the following additive system with a time-delay $\tau=2$,

$$
\begin{aligned}
& y(t)=\left[q^{-1}+0.9 q^{-2}\right] f(u(t))+\left[1-0.2 q^{-1}\right] g(y(t-3))+v(t), \\
& f\left(u(t)=u(t)-1+e^{u(t)},\right. \\
& \begin{aligned}
g(y(t-3))= & y(t-3)+\frac{m_{1}+m_{2}}{2} \operatorname{sgn}(y(t-3))+\frac{m_{1}-m_{2}}{2} \operatorname{sgn}\left(y^{2}(t-3)\right) \\
= & y(t-3)+\frac{0.4+0.2}{2} \operatorname{sgn}(y(t-3))+\frac{0.4-0.2}{2} \operatorname{sgn}\left(y^{2}(t-3)\right) \\
= & y(t-3)+0.3 \operatorname{sgn}(y(t-3))+0.1 \operatorname{sgn}\left(y^{2}(t-3)\right)
\end{aligned}
\end{aligned}
$$

the input $\{u(t)\}$ is taken as a persistent excitation signal sequence with zero mean and unit variance, and $\{v(t)\}$ is taken as a white noise sequence with zero mean and variance $\sigma^{2}=0.10^{2}$, the nonlinear input can be expressed as

$$
f(u(t))=c_{1} u(t)+c_{2} u^{2}(t)+c_{3} u^{3}(t)=2 u(t)+0.5 u^{2}(t)+0.17 u^{3}(t) .
$$

Assume $\mathrm{M}=6$, then we have 


$$
\begin{aligned}
\theta= & {\left[l_{1}, l_{2}, a_{1}, a_{2}, l_{5}, l_{6}, l_{1} d_{1}, l_{2} d_{1}, a_{1} d_{1}, a_{2} d_{1}, l_{5} d_{1}, l_{6} d_{1}, l_{1} d_{2}, l_{2} d_{2}, a_{1} d_{2}, a_{2} d_{2}, l_{5} d_{2},\right.} \\
& \left.l_{6} d_{2}, c_{1}, c_{2}, c_{3}, b_{2} c_{1}, b_{2} c_{2}, b_{2} c_{3}\right]^{T} \\
= & {[0,0,1,-0.2,0,0,0,0,0.3,-0.06,0,0,0,0,0.1,-0.02,0,0,2,0.5,0.17,1.8,0.45,0.153]^{T}, } \\
\varphi(t)= & y(t-1), y(t-2), y(t-3), y(t-4), y(t-5), y(t-6), \operatorname{sgn}(y(t-1)), \operatorname{sgn}(y(t-2)), \\
& \operatorname{sgn}(y(t-3)), \operatorname{sgn}(y(t-4)), \operatorname{sgn}(y(t-5)), \operatorname{sgn}(y(t-6)), \operatorname{sgn}\left(y^{2}(t-1)\right), \\
& \operatorname{sgn}\left(y^{2}(t-2)\right), \operatorname{sgn}\left(y^{2}(t-3)\right), \operatorname{sgn}\left(y^{2}(t-4)\right), \operatorname{sgn}\left(y^{2}(t-5)\right), \operatorname{sgn}\left(y^{2}(t-6)\right), \\
& \left.u(t-1), u^{2}(t-1), u^{3}(t-1), u(t-2), u^{2}(t-2), u^{3}(t-2)\right]^{T} .
\end{aligned}
$$

Applying the proposed method to estimate the parameters of this system, the parameter estimates and their errors are shown in Table 1, the parameter estimation errors $\delta:=\|\hat{\theta}-\theta\| /\|\theta\|$ versus t are shown in Figure 3.

From Table 1 and Figure 3, we can draw the following conclusions:

1. The parameter estimation errors be given by the RLS algorithm become smaller and smaller and go to zero with $t$ increasing.

2. Choose $\varepsilon=0.05$, we can compute the time-delay $\tau=2$ from (17).

3. In this example, when we choose a small $\varepsilon=0.005$, the irrelevant $l_{6} d_{1}$ will not be picked out. On the other hand, when we choose a big $\varepsilon=0.1$, we will regard the parameters $a_{2} d_{1}, a_{1} d_{2}$ and $a_{2} d_{2}$ as irrelevant parameters.

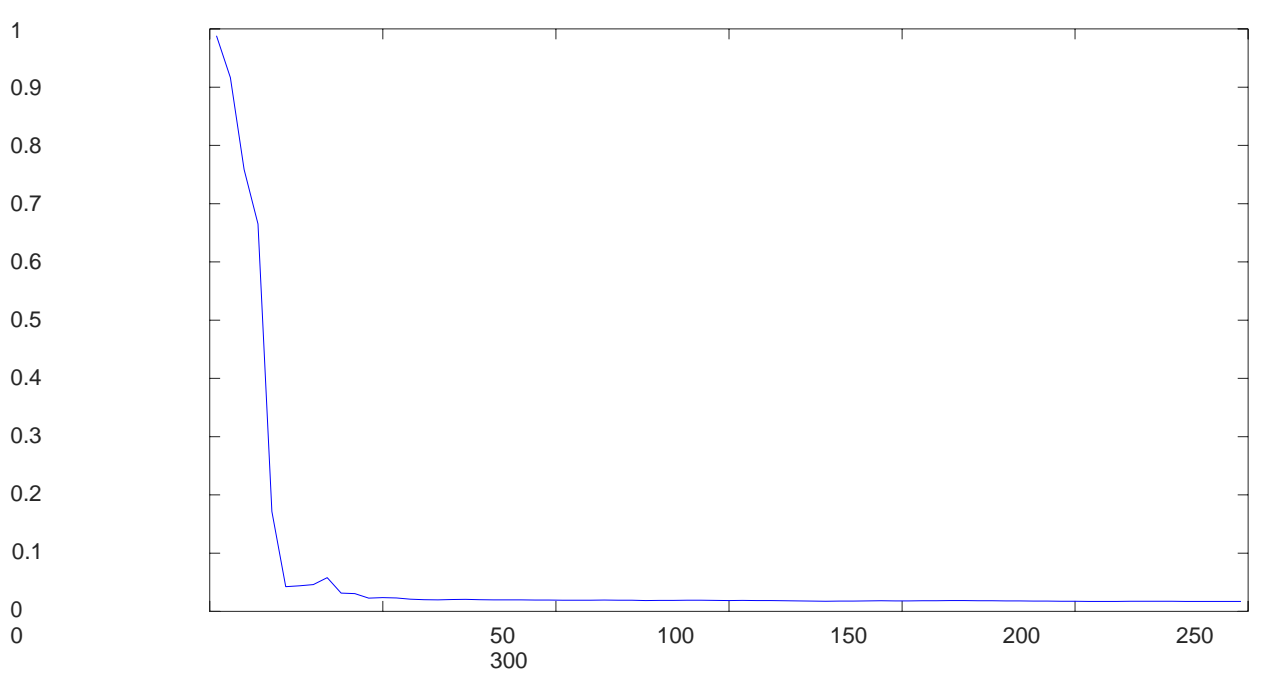

Figure 3. The Parameter Estimation Errors $\delta$ Versus $t$

\section{Conclusions}

This paper presents an identification method for nonlinear additive systems with unknown time- delay. By using the key term separation principle, the model of the nonlinear system can be turned into an identification model. Then the unknown timedelay and parameters can be estimated simulta- neously by a redundant parameter based recursive least squares algorithm. Though this method can estimate the time-delay, it also increases the number of the unknown parameters. In the future, we will find a method which cannot only estimate the time-delay but also can keep the number of the unknown parameters unchanged. 
Table 1. The RLS Estimates and Errors

\begin{tabular}{|c|c|c|c|c|c|c|c|c|}
\hline$t$ & 10 & 50 & 100 & 150 & 200 & 250 & 300 & True Values \\
\hline$l_{l}$ & 0.06426 & 0.00974 & 0.00478 & 0.00295 & 0.00472 & 0.00329 & 0.00311 & 0.00000 \\
\hline$l_{2}$ & -0.29939 & -0.00388 & -0.00290 & 0.00040 & -0.00216 & -0.00032 & -0.00018 & 0.00000 \\
\hline$a_{1}$ & 1.14728 & 1.01024 & 1.00266 & 1.00053 & 1.00150 & 1.00028 & 1.00001 & 1.00000 \\
\hline$a_{2}$ & -0.60532 & -0.20678 & -0.20315 & -0.20201 & -0.20429 & -0.20296 & -0.20310 & -0.20000 \\
\hline$l_{5}$ & 0.51674 & 0.00686 & 0.00470 & 0.00118 & 0.00331 & 0.00130 & 0.00081 & 0.00000 \\
\hline$l_{6}$ & -0.13896 & -0.01210 & -0.00252 & 0.00047 & -0.00178 & -0.00005 & -0.00003 & 0.00000 \\
\hline$l_{l} d_{l}$ & 0.27634 & -0.01236 & -0.00609 & -0.00769 & -0.00752 & -0.00771 & -0.00875 & 0.00000 \\
\hline$l_{2} d_{l}$ & -0.01625 & -0.01115 & 0.00215 & 0.00201 & 0.00249 & 0.00100 & 0.00262 & 0.00000 \\
\hline$a_{1} d_{1}$ & 0.42000 & 0.29191 & 0.30379 & 0.30351 & 0.29729 & 0.29553 & 0.29363 & 0.30000 \\
\hline$a_{2} d_{1}$ & 0.36188 & -0.06880 & -0.06407 & -0.05477 & -0.05561 & -0.05762 & -0.06012 & -0.06000 \\
\hline$l_{5} d_{1}$ & 0.12296 & 0.00408 & -0.00086 & -0.00733 & -0.00294 & -0.00127 & -0.00386 & 0.00000 \\
\hline$l_{6} d_{l}$ & -0.22369 & 0.01621 & -0.00319 & -0.00636 & 0.00244 & 0.00743 & 0.00734 & 0.00000 \\
\hline$l_{1} d_{2}$ & -0.00099 & & & & 0.00075 & & & \\
\hline$l_{2} d_{2}$ & -0.00174 & 0.00162 & 0.00148 & 0.00146 & 0.00130 & 0.00138 & 0.00131 & 0.00000 \\
\hline$a_{1} d_{2}$ & -0.09112 & 0.08472 & 0.07737 & 0.07617 & 0.06822 & 0.07221 & 0.06882 & 0.10000 \\
\hline$a_{2} d_{2}$ & 0.07455 & -0.06932 & -0.06330 & -0.06232 & -0.05581 & -0.05908 & -0.05630 & -0.02000 \\
\hline$l_{5} d_{2}$ & -0.00083 & 0.00077 & 0.00070 & 0.00069 & 0.00062 & 0.00066 & 0.00063 & 0.00000 \\
\hline$l_{6} d_{2}$ & -0.00166 & & 0.00141 & 0.00139 & 0.00124 & 0.00131 & 0.00125 & 0.00000 \\
\hline$c_{1}$ & 0.73647 & 1.97470 & 1.97762 & 1.98484 & 1.98396 & 1.98998 & 1.99020 & 2.00000 \\
\hline$c_{2}$ & -0.36542 & 0.48982 & 0.51195 & 0.51029 & 0.50303 & 0.50328 & 0.50443 & 0.50000 \\
\hline$c_{3}$ & 0.10370 & 0.17074 & 0.18081 & 0.17869 & 0.17576 & 0.17439 & 0.17433 & 0.17000 \\
\hline$c_{1} b_{2}$ & 0.68129 & 1.77670 & 1.78737 & 1.79249 & 1.79144 & 1.79548 & 1.79531 & 1.80000 \\
\hline$c_{2} b_{2}$ & -0.29918 & 0.44552 & 0.45871 & 0.46324 & 0.46360 & 0.46345 & 0.45715 & 0.45000 \\
\hline$c_{3} b_{2}$ & 0.02654 & 0.15436 & 0.15846 & 0.16003 & 0.15863 & 0.15898 & 0.15663 & 0.15300 \\
\hline$\delta(\%)$ & 75.92704 & 2.40341 & 2.00642 & 1.91751 & 1.85306 & 1.78613 & 1.75178 & \\
\hline
\end{tabular}

\section{Acknowledgment}

This work was supported by the National Natural Science Foundation of China (No. 61403165) and the Project of Philosophy and Social Science Research in Colleges and Universities in Jiangsu Province (No. 2014SJD381).

\section{References}

[1] E.W. Bai, "Identification of nonlinear additive FIR systems", Automatica, vol. 41, no. 7, (2005), pp. 1247-1253.

[2] E.W. Bai and K.S. Chan, "Identification of an additive nonlinear system and its applications in generalized Hammerstein models", Automatica, vol. 44, no. 2, (2008), pp. 430-436.

[3] E.W. Bai, "Identification of linear systems with hard input nonlinearities of known structure", Automatica, vol. 38, no. 5, (2002), pp. 853-860.

[4] F. Ding, X.H. Wang, Q.J. Chen and Y.S. Xiao, "Recursive least squares parameter estimation for a class of nonlinear systems based on the model decomposition", Circuits, Systems and Signal Processing, doi: 10.1007/s00034-015-0190-6, vol. 35, (2016).

[5] Q.H. Lin, X. Chen and J. Pena, "A smoothing stochastic gradient method for composite optimization", Optimization Methods and Software, vol. 29, no. 6, (2014), pp. 1281-1301.

[6] D.Q. Wang, T. Shan and R. Ding, "Data filtering based stochastic gradient algorithms for multivariable CARAR-like systems", Mathematical Modelling and Analysis, vol. 18, no. 3, (2013), pp. 374-385.

[7] C. Wang and T. Tang, "Recursive least squares estimation algorithm applied to a class of linear-inparameters output error moving average systems", Applied Mathematics Letters, vol. 29, (2014), pp. 3641.

[8] F. Ding, "System Identification—New Theory and Methods", Science Press, Beijing, (2013). 
[9] Y. Liu and E.W. Bai, "Iterative identification of Hammerstein systems", Automatica, vol. 43, no. 2, (2007), pp. 346-354.

[10] J. V"or"os, "Modeling and identification of systems with backlash", Automatica, vol. 46, no. 2, (2010), pp. 369-374.

[11] J. Chen and Y.X. Ni, "Parameter identification methods for an additive nonlinear system", Circuits, Systems and Signal Processing, vol. 33, no.10, (2014), pp. 3053-3064.

[12] Y. Rochdi and F. Giri, "Identification of block-oriented systems in the presence of nonparametric input nonlinearities of switch and backlash types", Automatica, vol. 46, no. 5, (2010), pp. 864-877.

[13] Y. Liu and E.W. Bai, "Iterative identification of Hammerstein systems", Automatica, vol. 43, no. 2, (2007), pp. 346-354.

[14] J. Chen, X.L. Lu and R. Ding, "Parameter identification of systems with preload nonlinearities based on the finite impulse response model and negative gradient search", Applied Mathematics and Computation, vol. 219, no. 5, (2012), pp. 2498-2505.

[15] J. V"or"os, "Modeling and parameter identification of systems with multi-segment piecewise-linear Characteristics", IEEE Transactions on Automatic control, vol. 47, no. 1, (2002), pp. 184-188.

[16] J. Chen, X.P. Wang and R.F. Ding, "Gradient based estimation algorithm for Hammerstein systems with saturation and dead-zone nonlinearities", Applied Mathematical Modelling, vol. 36, 1, (2012), pp. 238243.

[17] R. Jacob, N. Alexander and L. Lennart, "Nonlinear system identification via direct weight optimization", Automatica, vol.41, no. 3, (2005), pp. 475-490.

[18] B. Yu and H. Fang, "Identification of Hammerstein output-error systems with two-segment nonlinearities algorithm and applications", Control and Intelligent Systems, vol. 38, no. 4, (2010), pp. 194- 201.

[19] Y. Gu. F. Ding and J.H. Li, "States based iterative parameter estimation for a state space model with multi-state delays using decomposition”, Signal Processing, vol. 106, (2015), pp. 294-300.

[20] Y. Gu, F. Ding and J.H. Li, "State filtering and parameter estimation for linear systems with d-step statedelay”, IET Signal Processing, vol. 8, no. 6, (2014), pp. 639-646.

[21] L. Xie, H.Z. Yang and B. Huang, "FIR model identification of multirate processes with random delays using EM algorithm”, AIChE Journal, vol. 59, no. 11, (2013), pp. 4124-4130. 